\title{
Condições de trabalho autorreferida por profissionais de saúde durante a pandemia
} da Covid-19: Revisão integrativa

\author{
Self-reported working conditions by health professionals during the Covid-19 pandemic: \\ Integrative review
}

Condiciones de trabajo autorreferidas por profesionales de la salud durante la pandemia del Covid-19: Revisión integrativa

\section{Resumo}

Objetivo: Descrever as evidências cientificas disponiveis na literatura nacional e internacional sobre as condições de trabalho dos profissionais de saúde durante a pandemia de Covid-19. Método: revisão integrativa, realizada em seis bases de dados eletrônicas, sendo base de dados em Enfermagem, Literatura Latino-Americana e do Caribe em Ciências da Saúde, Medical Literature Analysis and Retrieval System Online, National Library of Medicine, SciVerse Scopus e Web of Science no período de junho e julho de 2020. Resultados: dos 596 artigos encontrados, foram selecionados apenas 02 que responderam a questão de pesquisa e as principais condições de trabalho evidenciadas foram em relação as reorganizações dos setores dentro da Instituição de saúde para o atendimento a pacientes acometidos por Covid-19, excesso na carga horária de trabalho, uso de equipamentos de proteção individual por muito tempo e desconfortáveis e situações geradoras de exaustão física e emocional. Conclusão: as condições de trabalho continuam sendo pautafoi agravada na pandemia e necessitam de ações públicas especificas.

Palavras-chave: Condições de trabalho; Enfermagem; Covid-19.

\begin{abstract}
Objective: To describe the scientific evidence available in the national and international literature on the working conditions of health professionals during the Covid-19 pandemic. Method: integrative review, carried out in six electronic databases, being the database in Nursing, Latin American and Caribbean Literature in Health Sciences, Medical Literature Analysis and Retrieval System Online, National Library of Medicine, SciVerse Scopus and Web of Science in the period of June and July 2020. Results: of the 596 articles found, only 02 were selected that answered the research question and the main working conditions evidenced were in relation to the reorganizations of the sectors within the Health Institution for the care of patients affected by Covid-19, excess load working hours, use of personal protective equipment for a long time and uncomfortable and situations that generate physical and emotional
\end{abstract}


exhaustion. Conclusion: working conditions continue to be aggravated in the pandemic and require specific public actions.

Keywords: Work conditions; Nursing; Covid-19.

\section{Resumen}

Objetivo: Describir la evidencia científica disponible en la literatura nacional e internacional sobre las condiciones laborales de los profesionales de la salud durante la pandemia Covid-19. Método: revisión integrativa, realizada en seis bases de datos electrónicas, siendo la base de datos en Enfermería, Literatura Latinoamericana y Caribeña en Ciencias de la Salud, Sistema de Análisis y Recuperación de Literatura Médica en Línea, Biblioteca Nacional de Medicina, SciVerse Scopus y Web of Science en el período de Junio y julio de 2020. Resultados: de los 596 artículos encontrados, solo se seleccionaron 02 que respondieron la pregunta de investigación y las principales condiciones laborales evidenciadas fueron en relación a las reorganizaciones de los sectores dentro de la institución de salud para la atención de pacientes afectados por Covid-19, sobrecarga laboral horas, uso de equipo de protección personal durante un tiempo prolongado e incómodo y situaciones que generan agotamiento físico y emocional. Conclusión: las condiciones laborales continúan agravándose en la pandemia y requieren acciones públicas específicas.

Palabras clave: Condiciones laborales; Enfermería; Covid-19.

\section{Introdução}

A COVID-19 é a doença infecciosa causada pelo coronavírus 2 (Sars-Cov-2), caracterizada pela síndrome respiratória aguda grave (WHO, 2020). O ser humano infectado por essa doença pode apresentar-se de forma assintomático ou sintomático, com sinais e sintomas de tosse, febre, mal-estar, dispneia e podendo evoluir para casos mais graves que requer cuidados hospitalares, incluindo necessitar do uso de ventilação mecânica (CDC, 2020). O novo coronavírus é uma doença altamente contagiosa, que ocasiona risco para a população, se espalha de forma muito rápida e, o aumento do número de contágios a elevou ao estado de pandemia (WHO, 2020; Zhan et al, 2020).

$\mathrm{O}$ alto percentual dos primeiros acontecimentos da patologia ocasionada pelo coronavírus, ocorreram no primeiro epicentro da pandemia, na cidade de Wuhan, capital da província da China Central, tendo em vista a relação da exposição ocupacional ou ambiental, uma vez que a provável origem da contaminação foi referente a um mercado de frutos do mar de Huanan, que manuseavam animais vivos. Contudo, de acordo com Li et al (2020), houve um aumento exponencial no número de casos não vinculados a tais condições constatando a transmissão de pessoa para pessoa. Fato que requereu imediatas medidas para controle da disseminação da infecção.

Neste contexto global, emergências sanitárias tornam-se necessárias para o enfrentamento da pandemia do novo coronavírus por meio de ações de precauções voltadas para a população ou para grupos com maior risco de contaminação. Autores como Jackson Filho et al (2020) e Silva, Machado, Oliveira et al (2020) referem a abrangência a proteção da saúde aos trabalhadores, em particular, dos profissionais da área da saúde, em relação a organização dos ambientes de trabalho para prevenir o adoecimento.

De acordo com Ribeiro et al (2020), os profissionais de saúde, compostos por distintas categorias, estão diretamente implicados no atendimento às pessoas infectadas pela Covid-19 e, por este motivo, compõem um grupo de risco específico para a infecção. Dentre estes, destaca-se a enfermagem, que estão na linha de frente de enfrentamento ao coronavírus, e atuam em contato direto com pessoas face-a-face, com exigência da assistência para com todos os pacientes, em especial aos graves. Corroborando com Teixeira et al (2020), estes profissionais atuam diretamente com pacientes infectados, o que faz com que recebam uma alta carga viral (milhões de partículas de vírus), consequentemente maior exposição a contrair a Covid-19.

Frente a essas considerações, nota-se que tais profissionais, em suas atividades laborais podem estar sobre diversas condições durante a execução do seu trabalho, que lhes permitam desenvolver suas funções de maneira segura ou não. Um dos grandes desafios dessa pandemia de Covid-19 é garantir a segurança e a proteção efetiva dos profissionais de saúde em um cenário de muitas dúvidas (Adalja, Toner \& Inglesby, 2020), considerando-se o insuficiente conhecimento sobre a etiologia, formas de tratar, controlar a doença e, principalmente, a sua alta transmissibilidade (Barreto et al, 2020). 
Ainda, tendo como sustentação a vivência profissional, pode-se observar que as transformações ocorridas nas condições de trabalho da equipe de saúde perpetuam atitude de inatividade diante do que é adequado, possibilitando, sofrimento psíquico e físico. Dessa maneira, é motivador a realização deste estudo, tendo em vista que, no momento em que se enfrenta uma nova doença, em especial a pandemia, se deve considerar todos os aspectos presentes que envolvem as condições de trabalho.

Por ser uma temática atual essa discussão é necessária para que se conheçam as condições de trabalho em que os profissionais de saúde/enfermagem lidam diariamente frente ao contexto da pandemia por Covid-19, seja a nível nacional e internacional. Assim, o objetivo desta pesquisa é descrever as evidências cientificas disponiveis na literatura nacional e internacional sobre as condições de trabalho dos profissionais de saúde durante a pandemia de Covid-19.

\section{Metodologia}

O estudo trata-se de revisão integrativa. Este tipo de método reúne, avalia e sintetiza os resultados de pesquisas sobre uma temática específica (Mendes, Silveira e Galvão, 2008). A abordagem permite a combinação de vários métodos de estudo e diversos propósitos, que tem o potencial de resultar em conceitos, teorias ou problemas de saúde de importância para a área da enfermagem (Whittemore \& Knafl, 2005).

As etapas relacionadas ao método de estudo são compostas por seis: Etapa I - identificação do tema e/ou questão de pesquisa; Etapa II - estabelecimento de critérios para inclusão e exclusão dos estudos e busca sistematizada na literatura; Etapa III - definição das informações a serem extraídas dos estudos selecionados conforme a questão norteadora da revisão; Etapa IV - avaliação dos estudos incluídos na revisão; Etapa V - interpretação dos resultados encontrados; Etapa VI - síntese do conhecimento (Soares et al, 2014).

A etapa de seleção dos estudos ocorreu por meio de busca eletrônica a artigos originais que respondessem à pergunta norteadora. Para a formulação da pergunta de pesquisa, foi utilizado o acrônimo PICO, em que P corresponde ao paciente ou população, I é a intervenção, $\mathrm{C}$ a comparação ou controle e $\mathrm{O}$ é o desfecho ou resultado. $\mathrm{O}$ uso dessa estratégia para formular a questão de pesquisa na condução de métodos de revisão possibilita a identificação de palavras-chave, as quais auxiliam na localização de estudos primários relevantes nas bases de dados (Stillwell et al, 2010). Assim, a questão de pesquisa norteadora foi: Quais as evidências científicas em relação as condições de trabalho em que os profissionais de saúde estão submetidos no contexto da pandemia por Covid-19? A depender do método de revisão, não se emprega todos os elementos da estratégia PICO (Garcia et al, 2016).

Os critérios de inclusão que compuseram a seleção dos artigos foram: artigos publicados e disponíveis na íntegra advindos de todos os níveis de evidência, em português, inglês e espanhol, publicados de 2019 a julho de 2020, que respondessem à pergunta de investigação. $\mathrm{O}$ recorte temporal pautou-se na atualidade do contexto estudado. Foram excluídos trabalhos de reflexão quanto a manuais/protocolos, relato de experiência e carta aos editores.

As bases de dados eletrônicas acessadas foram: Base de dados em Enfermagem (BDENF), Literatura LatinoAmericana e do Caribe em Ciências da Saúde (LILACS), Medical Literature Analysis and Retrieval System Online (MEDLINE), National Library of Medicine (PUBMED), SciVerse Scopus e Web of Science. Os descritores utilizados foram submetidos na Biblioteca Virtual em Saúde, Descritores em Ciências da Saúde (DeCS) e no Medical Subject Heading (MeSH), utilizando para todos os cruzamentos o operador booleano AND: Covid-19 AND enfermagem; Covid-19 AND enfermagem AND condições de trabalho; Ambiente de trabalho AND coronavirus. No Quadro 1 abaixo, pode-se observar o delineamento realizado nesta etapa da pesquisa. 
Research, Society and Development, v. 10, n. 6, e49310615867, 2021

(CC BY 4.0) | ISSN 2525-3409 | DOI: http://dx.doi.org/10.33448/rsd-v10i6.15867

Quadro 1: Estratégia de busca nas bases de dados.

\begin{tabular}{|l|l|}
\hline Base de dados & Estratégia de busca \\
\hline BDENF & $\begin{array}{l}\text { Covid-19 AND enfermagem } \\
\text { Covid-19 AND enfermagem AND condições de trabalho } \\
\text { Ambiente de trabalho AND coronavirus }\end{array}$ \\
\hline LILACS & $\begin{array}{l}\text { Covid-19 AND enfermagem } \\
\text { Covid-19 AND enfermagem AND condições de trabalho } \\
\text { Ambiente de trabalho AND coronavirus }\end{array}$ \\
\hline MEDLINE & $\begin{array}{l}\text { Covid-19 AND enfermagem } \\
\text { Covid-19 AND enfermagem AND condições de trabalho } \\
\text { Ambiente de trabalho AND coronavirus }\end{array}$ \\
\hline PUBMED & $\begin{array}{l}\text { Covid-19 AND enfermagem } \\
\text { Covid-19 AND enfermagem AND condições de trabalho } \\
\text { Ambiente de trabalho AND coronavirus }\end{array}$ \\
\hline SciVerse Scopus & $\begin{array}{l}\text { Covid-19 AND enfermagem } \\
\text { Covid-19 AND enfermagem AND condições de trabalho } \\
\text { Ambiente de trabalho AND coronavirus }\end{array}$ \\
\hline Web of Science & $\begin{array}{l}\text { Covid-19 AND enfermagem } \\
\text { Covid-19 AND enfermagem AND condições de trabalho } \\
\text { Ambiente de trabalho AND coronavirus }\end{array}$ \\
\hline
\end{tabular}

Fonte: Base de dados e estratégia de busca selecionada. Rondonópolis, MT, Brasil (2020).

No Quadro 1, nota-se que foram utilizadas 06 bases de dados eletrônicas que possuem afinidade com temáticas da área da saúde. Percebe-se ainda, que houve a uniformidade nos cruzamentos durante a busca realizada.

Para a descrição do estudo, utilizou-se o fluxograma Preferred Reporting Items for Systematic Review and MetaAnalyses (PRISMA) para sistematizar o processo de elegibilidade dos estudos (Moher et al, 2009). Este modelo foi utilizado para nortear a busca e a seleção dos estudos, representado a seguir na Figura 1. 
Figura 1: Fluxograma de seleção dos estudos quanto ao processo de identificação, triagem e elegibilidade dos estudos. Rondonópolis, MT, Brasil, 2020.

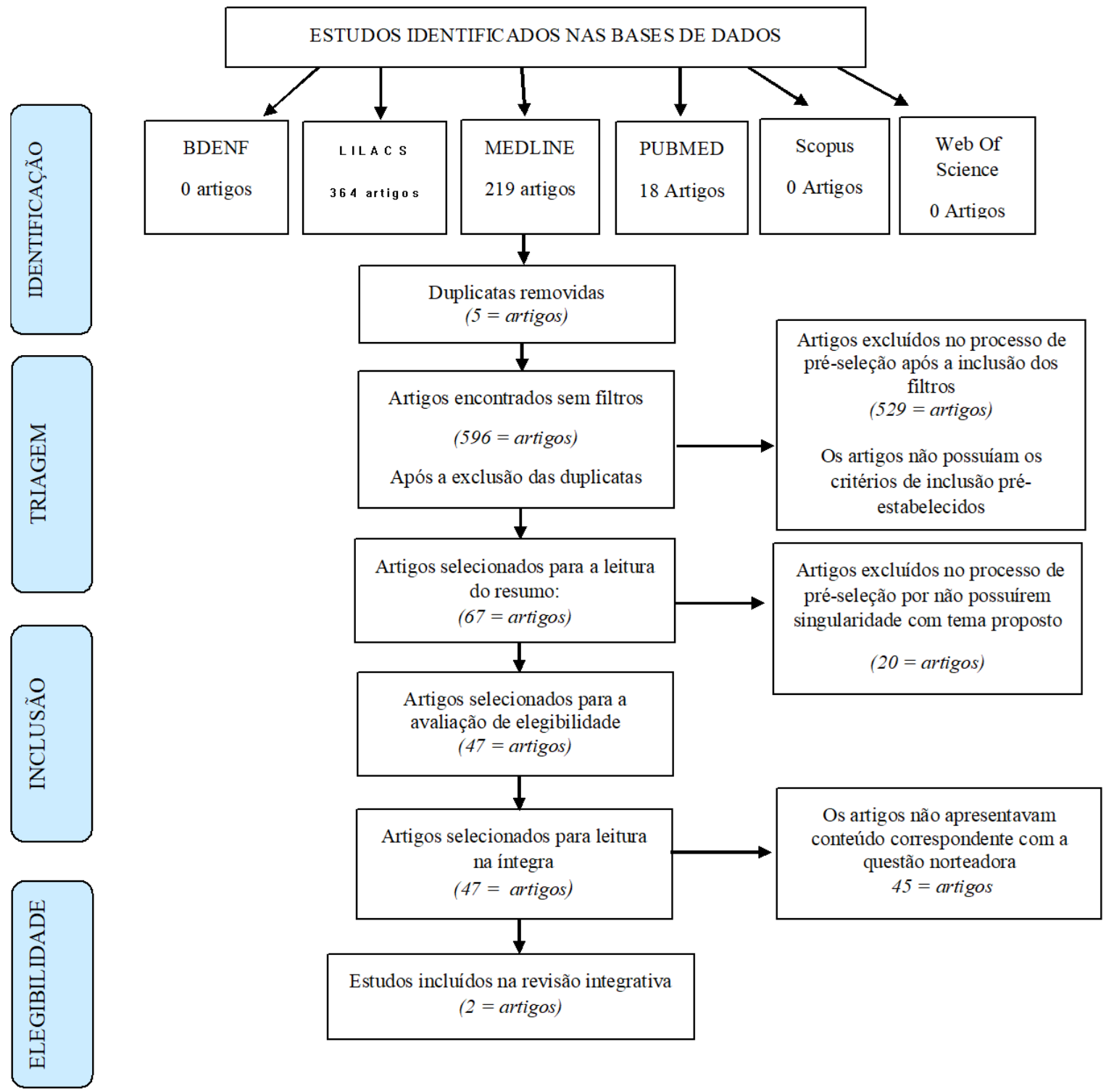

Fonte: Autores.

A figura acima observa-se o detalhamento das buscas realizadas nas plataformas de base de dados citadas, as duplicatas de estudo encontradas, os critérios de inclusão e exclusão de artigos e o resultado daqueles que compuseram a amostra do presente estudo.

A coleta de dados ocorreu nos meses de junho e julho de 2020, foram inseridos nas bases eletrônicas os descritores, previamente, respeitando os critérios de inclusão e exclusão. Após esse processo os artigos foram selecionados por intermédio da leitura dos títulos somente aqueles que apresentavam correlação com o tema principal.

Posteriormente à análise dos títulos, os artigos foram antepostos por meio de uma leitura criteriosa de seus resumos e aqueles que apresentavam singularidade com o tema proposto pela pergunta norteadora foram escolhidos para leitura na íntegra, e para a consolidação realizou-se leitura completa e avaliação dos artigos. Para fidedignidade do estudo foi realizada dupla checagem entre pesquisadores de maneira independente. 
A análise dos estudos selecionados foi realizada de forma descritiva, nesta perspectiva, os achados permitiram responder à questão quanto às condições de trabalho dos profissionais de saúde no contexto atual da pandemia.

\section{Resultados}

A amostra da revisão integrativa foi composta de dois estudos, ambos publicados no ano de 2020. A partir dos achados, os resultados foram sistematizados em quadros.

O Quadro 2 a seguir, contém informações pertinentes aos artigos, sendo apresentados a base de dados, autores e ano de publicação, título do artigo, periódico de divulgação e local de estudo, que apontam explicações relativas ao problema de pesquisa.

Quadro 2: Caracterização dos estudos conforme a base de dados, autores e ano, título, periódico e local de estudo. Rondonópolis, MT, Brasil, 2020.

\begin{tabular}{|l|l|l|l|l|l|}
\hline $\begin{array}{c}\text { N. } \\
\text { Art. }\end{array}$ & $\begin{array}{c}\text { Base de } \\
\text { dados }\end{array}$ & \multicolumn{1}{|c|}{$\begin{array}{c}\text { Autores e } \\
\text { Ano }\end{array}$} & \multicolumn{1}{|c|}{ Título } & $\begin{array}{c}\text { Periódico } \\
\text { estudo }\end{array}$ \\
\hline A1 & Lilacs & $\begin{array}{l}\text { Zhang et al, } \\
2020 .\end{array}$ & $\begin{array}{l}\text { The Psychological Change Process of } \\
\text { Frontline Nurses Caring for Patients with } \\
\text { COVID-19 during Its Outbreak. }\end{array}$ & $\begin{array}{l}\text { Issues in Mental } \\
\text { Health Nursing }\end{array}$ & China \\
\hline A2 & Lilacs & $\begin{array}{l}\text { Liu et al, } \\
2020 .\end{array}$ & $\begin{array}{l}\text { The experiences of health-care providers } \\
\text { during the COVID-19 crisis in China: a } \\
\text { qualitative study. }\end{array}$ & $\begin{array}{l}\text { The lancet Global } \\
\text { Health }\end{array}$ & China \\
\hline
\end{tabular}

Fonte: Dados da pesquisa.

Os estudos encontrados são internacionais, ambos realizados na China e divulgados em 2020. A seguir, no quadro 3 apresenta-se a síntese dos estudos analisados. 
Quadro 3: Caracterização dos estudos de acordo com autores, objetivo, tipo de estudo, população do estudo e principais resultados referentes as condições de trabalho durante a pandemia. Rondonópolis, MT, Brasil, 2020.

\begin{tabular}{|c|c|c|c|c|}
\hline N. & Objetivo & $\begin{array}{l}\text { Tipo de } \\
\text { estudo }\end{array}$ & $\begin{array}{l}\text { População do } \\
\text { estudo }\end{array}$ & $\begin{array}{l}\text { Principais resultados referentes às condições de trabalho durante a } \\
\text { pandemia }\end{array}$ \\
\hline A1 & $\begin{array}{l}\text { Identificar o processo } \\
\text { de mudança } \\
\text { psicológica } \\
\text { enfermeiras } \\
\text { atuavam no epicentro } \\
\text { do surto de Covid-19. }\end{array}$ & $\begin{array}{l}\text { Descritivo } \\
\text { Qualitativo }\end{array}$ & Enferr & $\begin{array}{l}\text { - Enfrentamento de escassez de enfermeiras para atuar no epicentro; } \\
\text { - Ambiente e colegas de trabalho desconhecidos; } \\
\text { - Medo de contaminação; } \\
\text { - Uso de Equipamentos de Proteção Individual (EPIs) desconfortáveis e } \\
\text { medo de não estarem corretos; } \\
\text { - Características presentes como medo, temor do desconhecido, a } \\
\text { incerteza sobre o desfecho da doença e as situações incontroláveis, } \\
\text { ansiedade, somatização, compulsividade, irritação; } \\
\text { - Fluxo de trabalho complexo; } \\
\text { - Exaustão emocional, isolamento interpessoal, resiliência frágil; } \\
\text { - Reconhecimento de líderes de enfermagem quanto as respostas } \\
\text { psicológicas, condições de trabalho; } \\
\text { - Ajuste do tempo de cada turno de } 6 \text { horas para } 4 \text { horas para } \\
\text { enfermagem. } \\
\text { - Treinamentos para a equipe. }\end{array}$ \\
\hline $\mathrm{A} 2$ & $\begin{array}{l}\text { Descrever as } \\
\text { experiências desses } \\
\text { profissionais de saúde } \\
\text { nos estágios iniciais } \\
\text { do surto. }\end{array}$ & Qualitativo & $\begin{array}{l}\text { Enfermeiros e } \\
\text { médicos }\end{array}$ & $\begin{array}{l}\text { - Adaptação a um novo ambiente de trabalho; } \\
\text { - Colegas de trabalho desconhecidos e de várias especialidades; } \\
\text { - Equipe com dificuldade quanto a comunicação; } \\
\text { - Medo de ser infectado e infectar colegas e familiares; } \\
\text { - Uso de EPIs desconfortáveis e por longos períodos; } \\
\text { - Carga horária de trabalho demasiada; } \\
\text { - Exaustão física e emocional; } \\
\text { - Reorganização da estrutura física; } \\
\text { - Limitação de números de prestadores de cuidados de saúde para } \\
\text { garantir a proteção; } \\
\text { - Redução do tempo para as necessidades de alimentação e eliminação; } \\
\text { - Apoio social, logístico do hospital e incentivo dos colegas o que } \\
\text { proporcionou segurança; } \\
\text { - Criação de grupo online de aconselhamento psicológico. }\end{array}$ \\
\hline
\end{tabular}

Fonte: Dados da pesquisa.

Os trabalhos apresentam características semelhantes. A população de estudo foram os profissionais de saúde, em especial, a equipe de enfermagem.

Evidenciam a mesma abordagem metodológica, de natureza qualitativa e expressam as condições de trabalho dos profissionais de saúde/enfermagem durante a pandemia de Covid-19. As principais condições de trabalho referenciadas nos estudos foram: excesso na carga horária de trabalho, uso de Equipamentos de Proteção Individual (EPIs) desconfortáveis, situação geradora de exaustão física e emocional, ambiente e colegas de trabalho desconhecidos; medo de contaminação e outros. 


\section{Discussão}

Sobre os fenômenos validados nos artigos analisados, observou-se que foram contemplados aspectos semelhantes, que modificaram as condições de trabalho. Além disso, foram destacadas nos estudos as experiências exitosas realizadas nas instituições pesquisadas que favoreceram os profissionais de saúde/enfermagem em relação às condições de trabalho.

De acordo com o artigo 1, com o aparecimento repentino da doença, os enfermeiros foram encaminhados para o epicentro da pandemia por escassez, atuando longe do seu local habitual de serviço (Zhan et al, 2020). Complementando essa ideia, a pesquisa 2 refere a limitação do número de prestadores de saúde, tal situação influencia diretamente os cuidados destinados ao paciente. Estes profissionais possuem alto risco de serem infectados tanto devido à proximidade com os pacientes contaminados, quanto ao não recebimento de treinamentos e proteção suficiente ao recebimento (ONU, 2020).

Os artigos destacam como aspectos dificultadores das condições de trabalho, o ambiente. O estudo 1 retrata a atuação dos profissionais de saúde/enfermagem em ambiente desconhecido de trabalho devido o deslocamento para locais diferentes de sua origem, associado ao exercício profissional com colegas desconhecidos. A pesquisa 2, destaca que a maioria dos profissionais que integravam a nova equipe de saúde era composto de colegas de trabalho desconhecidos, com especialidades diferentes, de outras províncias, o que dificultou a comunicação em equipe, consequentemente tornou-se a adaptação a um novo ambiente de trabalho um desafio. Os autores pontuam como um desafio adicional neste novo contexto de trabalho estressante, a inexperiência de alguns profissionais de saúde que estavam na linha de frente no cuidado a pacientes com doenças infecciosas (Liu et al, 2020). Convém destacar que o simples fato de se ter que exercer suas atividades laborais em um local desconhecido por si só gera estranheza e desconforto e isso é acentuado em situação de pandemia, frente a uma doença pouco conhecida.

Diante da configuração mundial, o desafio em atuar em uma atividade não habitual, pode vir carregado por sentimentos de medo. Características estas, apresentadas nos artigos encontrados, como refere o estudo1 em que o medo de contaminação pelo novo coronavírus é fator influenciador na condição de trabalho (Zhan et al, 2020). A pesquisa 2 refere medo de se infectar e de infectar seus pares, sejam eles familiares ou colegas de expediente, por ser uma patologia de fácil disseminação e em constante progressão mundial (Liu et al, 2020). No atual contexto da pandemia os profissionais de saúde enfrentam o medo de se infectarem ou transmitirem a infecção aos membros da família (Fiocruz, 2020 e Oliveira, Araujo e Migueis, 2020).

Temáticas abordadas de consenso nos artigos foi o uso de EPIs. Em um artigo o fato se deve por serem desconfortáveis, pesados, causando falta de ar, aperto no peito e dificuldade de visualização devido ao uso do óculos de proteção, associado a isso, o medo de não estarem utilizando de maneira correta (Zhan et al, 2020). Em outro estudo, o uso de EPIs também foi destacado como sendo desconfortáveis e sua utilização por várias horas gera um grande desafio de resistência física e atuação profissional. O calor excessivo favorece o aumento da transpiração, o qual molhava não só suas vestes, como também o protetor ocular, que ficava translúcido, dificultando assim visualizar os próprios colegas de trabalho e até a leitura dos rótulos dos medicamentos (Liu et al, 2020).

$\mathrm{Na}$ atual conjuntura de pandemia, foi garantida a distribuição de forma suficiente e adequada aos profissionais de saúde/enfermagem. De acordo com a legislação trabalhista brasileira via Norma Regulamentadora de Segurança e Saúde no Trabalho em Serviços de Saúde 32, preconiza-se a obrigatoriedade do empregador em oferecer ao trabalhador EPI em quantidade suficiente. Esses equipamentos devem ser garantidos o imediato fornecimento ou reposição para a realização segura das atividades laborais, afim de reduzir risco de contaminação nos profissionais de saúde, sendo esta condição essencial para os profissionais de saúde na prestação dos cuidados (Brasil, 2006).

Ainda o estudo 2 reforça a ideia de utilização adequada desses dispositivos de proteção com a necessidade de providenciar treinamento a todos os membros da equipe multiprofissional (Liu et al, 2020). O fornecimento de EPI adequado, 
em número suficiente, com treinamento de acordo com protocolos e aumento da conscientização sobre a proteção pessoal, pode contribuir para a redução do risco de infecção em profissionais de saúde (Wang et al, 2020), além de ser essencial para o gerenciamento do estresse causado pelas atividades laborais (Huang et al, 2020).

Entretanto, na pesquisa 2 foi evidenciado, a fim de reduzir o uso de suprimentos de EPIs, a redução do tempo para as necessidades de alimentação e eliminação (Liu et al, 2020). Estudo de Silva e Migueis (2020) sobre o risco enfrentados pela equipe de enfermagem frente ao coronavirus, revelou a reutilização dos EPIs. O risco de contaminação pela falta de EPIs bem como uso exacerbado desses equipamentos, em turnos de até 6 horas ininterruptas em unidades de terapias intensivas, com uso de fraldas, promove ansiedade no momento da desparamentação, ou seja, na retirada desses equipamentos, o que tem provocado um intenso sofrimento nestes profissionais, levando, inclusive, ao afastamento do trabalho, o que compromete, ainda mais, a qualidade do atendimento prestado aos pacientes (Teixeira et al, 2020).

Destacam Helioterio et al (2020), que esta é uma questão central uma vez que dificuldades no acesso e uso de EPIs adequados contribuem para aumentar a exposição do profissional de saúde/enfermagem ao coronavírus. A garantia de condições seguras para o exercício profissional, com as barreiras físicas adequadas proporcionadas pelos EPIs é o ponto inicial, a condição sine qua non para a atividade de trabalho. Essa garantia não pode ser flexibilizada ou improvisada em nenhuma circunstância.

Segundo os autores, é sabido que os profissionais de saúde convivem, cotidianamente, com condições de trabalho precárias, decorrentes de escassez de recursos e materiais ou de características da organização do trabalho em saúde (Helioterio et al, 2020). Neste sentido, sobressai o achado no estudo 2, em que é demasiada a carga horária de trabalho e associado a redução do tempo para as necessidades de alimentação e eliminação durante os plantões, levam ao intenso desgaste físico e emocional dos profissionais que atuam na linha de frente de enfrentamento ao Covid-19 (Liu et al, 2020). Demais autores como, Helioterio et al (2020) e Santana (2018) referem que extensas jornadas, ritmo intenso, trabalho em turnos, dificuldade para pausas e repouso, desvalorização profissional, conflitos interpessoais, entre outros fatores desencadeantes de desgastes físicos e psíquicos são condições desfavoráveis do trabalho. Embora essas dificuldades exacerbam-se em situação de crise, elas são frequentemente identificadas no trabalho em saúde. Notamos que o momento atual da pandemia ilumina problemas antigos, mas que não foram devidamente enfrentados no contexto brasileiro.

Ressaltam Barbosa et al (2020), que as equipes de saúde correm mais riscos de contraírem o coronavírus por estarem mais expostos e Moraes et al (2020) acrescentam que essa exposição traz uma série de danos, que vão além do adoecer. Envolve a preocupação com seus familiares, o conviver com suspensão de aulas dos filhos, a reorganização da forma de ir e vir assumindo assim, mais riscos e responsabilidades, que não faz parte do cotidiano normal.

É a categoria profissional mais afetada psicologicamente, uma vez que experimenta de fatores estressores já elencados como medo, temor do desconhecido, a incerteza sobre o desfecho da doença, a exaustão emocional, isolamento interpessoal, resiliência frágil e as situações incontroláveis como ansiedade, somatização, compulsividade, irritação, conforme descrito no artigo de Zhan et al (2020). O segundo artigo selecionado no estudo reforça tais características quando se refere que os profissionais de saúde/enfermagem que atuam no enfrentamento a pandemia de Covid-19 apresentam exaustão emocional e física. Autores como Oliveira, Araujo e Migueis (2020), retratam que o cenário tem se modificado devido aos inegáveis casos de coronavírus, e aspectos como insegurança, medo e incerteza permeiam a equipe de enfermagem sendo potenciais geradores de estresse. A insegurança propiciada pelo fator do vírus ser pouco estudado é uma das grandes preocupações da equipe, além disso, há o despreparo desses profissionais que estão no atendimento a esses pacientes.

Neste sentido, o reconhecimento dos gestores da enfermagem quanto às respostas psicológicas, condições de trabalho são fundamentais para qualidade da assistência ao paciente de qualquer patologia e situação clínica. $\mathrm{O}$ aporte na comunicação e 
nas condições de trabalho podem amenizar os problemas apresentados no contexto laboral, bem como, o aparecimento de patologias psicológicas.

É notório que o trabalho da enfermagem é complexo, diverso e multifacetado (Soares et al, 2020), e esta condição permanece na pandemia como retratado no artigo 1 , onde se refere que o fluxo de trabalho dos profissionais de saúde/enfermagem é complexo. Para Soares et al (2020) ao executar suas atividades, esses trabalhadores se expõem continuamente a uma série de riscos ocupacionais e as condições inadequadas no contexto laboral, são contribuintes as condições em que muitos trabalhadores de enfermagem vêm ao longo dos anos, ocasionando em adoecimento físico e mental.

Sustentado pela pesquisa 2, neste momento atual em que não há compreensão desse novo vírus, e o mesmo possui altíssimo potencial de transmissibilidade, patogenicidade e tentativas de tratamentos ineficazes, visto por se tratar de algo novo, os estudos clínicos continuam em busca a uma possível cura para Covid-19 (Liu et al, 2020), expondo-os ainda mais ao risco de contaminação por todas as condições laborais.

Em contrapartida, os artigos pesquisados demonstram contribuições significativas no momento pandêmico. $O$ artigo 1 reconhece apoio de líderes de enfermagem para as respostas psicológicas e condições de trabalho. Estas últimas se referem a realização de treinamentos a equipe, incluindo no processo, o cuidado de colocar e remover os EPIs com cautela, sem risco de contaminação, e outro fator foi o ajuste na carga horária de cada turno de 6 horas para 4 horas, possibilitando que mais enfermeiras deveriam participar do trabalho de isolamento da unidade, e enfatizam que os arranjos devem ser realizados conforme a cena da prática.

O estudo 2 destaca a criação de grupo online de aconselhamento psicológico para profissionais e pacientes. Obter apoio social, logístico do hospital e incentivo dos colegas, são aspectos que melhoram a segurança no ambiente de trabalho.

Diante desses resultados, são inegáveis a variedade de fatores que influenciam nas condições de trabalho dos profissionais da saúde perante a pandemia do novo coronavírus. Até mesmo o uso de EPI, que é uma conduta básica, pode gerar desconfortos físicos e psicológicos, privando os profissionais de suas necessidades básicas para evitar desperdícios dos materiais.

Contudo, este estudo possui limitações, visto que a temática é específica, extremamente atual, uma doença ainda pouco estudada e vivenciada nos ambientes de trabalho, assim explicando os estudos clínicos incipientes. Outra limitação evidenciada foi à escassez de trabalhos científicos na literatura, de modo que a mesma é comprovada pelo número reduzido de artigos selecionados para análise de discussão, sendo justificado pelo período da coleta de dados que iniciou juntamente com a pandemia.

\section{Conclusão}

Os profissionais da enfermagem são essenciais para o cuidado ao ser humano e tornam-se protagonistas perante a assistência ao paciente no enfrentamento da pandemia de Covid-19. Consequentemente suas condições de trabalho também estiveram mais evidentes e sofreram modificações no contexto estrutural, organizacional, relacional e em sua saúde psíquica e física.

Algumas mudanças de âmbito estrutural podem ser evidenciadas com as reorganizações dos setores dentro da Instituição de saúde para o atendimento a pacientes acometidos por Covid-19. Os profissionais de saúde/enfermagem foram realocados para outros locais e/ou setores requerendo em adaptação a um novo ambiente de trabalho, podendo ainda estar associado à inexperiência de cuidado.

Quanto a âmbito organizacional, destacam-se muitos aspectos como a carga de trabalho exaustiva, o uso do EPI por longo período de tempo, sendo o mesmo desconfortável para os profissionais que o utilizavam. Ainda, o fluxo de trabalho complexo e perigos decorrentes da exposição a pacientes de Covid-19. 
No aspecto relacional, ficou evidente o trabalhar com colegas desconhecidos, sem aproximação interpessoal, vivenciando o isolamento, a resiliência frágil. A exaustão emocional e física se faz evidentes.

Diante disso, nota-se que a situação das condições de trabalho dos profissionais de saúde/enfermagem não são as adequadas neste contexto da pandemia e tornou-se mais desfavorável, contudo, reflete uma condição antiga desses profissionais. Mesmo perante uma situação desconhecida, inesperada é importante ter a atenção pública com ações efetivas que possa adotar estratégicas de preservação da saúde em todos os aspectos referente às condições de trabalho dos profissionais de saúde/enfermagem atuantes na linha de frente ao combate a pandemia de Covid-19.

Espera-se que diante de toda situação vivenciada por esses profissionais em meio à pandemia, possam ter o reconhecimento, a valorização profissional da sociedade em geral e dos gestores públicos, e, contudo, condições dignas de trabalho. Recomenda-se que novos estudos sejam realizados nos diversos países, em diferentes contextos de atuação e com os diversos delineamentos de pesquisa para que se possa dar visibilidade ao trabalho dos profissionais de saúde, tendo em vista a melhoria dos ambientes de trabalho e a promoção da saúde física e mental.

\section{Referências}

Adalja, A. A., Toner, E. \& Inglesby, T. V. (2020). Priorities for the US health community responding to COVID-19. JAMA, 323 (14), 1343-4.

Barbosa, D. J., Gomes, M. P., Souza, F. B. A. \& Gomes, A. M. T. (2020). Fatores de estresse nos profissionais de enfermagem no combate à pandemia da COVID-19: Síntese de Evidências. Com. Ciências Saúde, 31, 31-47.

Barreto, M. L., Barros, A. J. D., Carvalho, M. S., Codeço, C. T., Hallal, P. R. C., Medronho, R. A. et al (2020). O que é urgente e necessário para subsidiar as políticas de enfrentamento da pandemia de COVID-19 no Brasil? Rev Bras Epidemiol, 23 (e200032).

Brasil, Ministério da Saúde. Secretaria de Atenção à Saúde (2006). Departamento de Ações Programáticas Estratégicas. Exposição a materiais biológicos. http://bvsms.saude.gov.br/bvs/publicacoes/protocolo_expos_mat_biologicos.pdf.

Centers for Disease Control and Prevention (CDC) (2020). Severe Outcomes Among Patients with Coronavirus Disease 2019 (COVID-19): United States, February 12-March 16, 2020. Morbidity and Mortality Weekly Report, 69 (12), 343-346.

Fundação Oswaldo Cruz (FIOCRUZ), Brasil (2020). Saúde mental e atenção psicossocial na pandemia Covid. https:/portal.fiocruz.br/documento/saudemental-e-atencao-psicossocial-na-pandemia-covid-19.

Garcia, A. K. A., Fonseca, L. F., Aroni, P. \& Galvão, C. M. (2016). Strategies for thirst relief: integrative literature review. Rev Bras Enferm., 69 (6), $1148-55$.

Helioterio, M. C., Lopes, F. Q. R. S., Sousa, C. C., Souza, F. O., Pinto, O. S., Sousa, F. N. F. et al (2020). Covid-19: Por que a proteção de trabalhadores e trabalhadoras da saúde é prioritária no combate à pandemia? Trab. educ. saúde, 18 (3).

Huang, L., Lin, G., Tang, L., Yu, L. \& Zhou, Z. (2020). Special attention to nurses’ protection during the COVID-19 epidemic. Critical Care 24 (120).

Jackson Filho, M. J., Assunção, A. A., Algranti, E., Garcia, E. G., Saito, C. A. \& Maeno, M. (2020). A saúde do trabalhador e o enfrentamento da COVID19. Rev. bras. saúde ocup. $45,17$.

Li, Q., Guan, X., Wu, P., Wang, X., Zhou, L., Tong, Y. et al (2020). Early Transmission Dynamics in Wuhan, China, of Novel Coronavirus-Infected Pneumonia. New England Journal of Medicine, 382 (13), 1199-1207.

Liu, Q., Luo, D., Haase, J. E., Guo, Q., Wang, X. Q., Liu, S. et al (2020). The experiences of health-care providers during the COVID-19 crisis in China: a qualitative study. The Lancet Global Health, $8(60)$.

Mendes, K. D. S., Silveira, R. C. C. P. \& Galvão, C. M. (2008). Revisão integrativa: método de pesquisa para a incorporação de evidências na saúde e na enfermagem. Texto Contexto Enferm, 17 (4), 758-64.

Moher, D., Liberati, A., Tetzlaff, J., Altman, D. G. (2009). Preferred reporting items for systematic reviews and meta-analyses: the PRISMA statement. Annal intern med., 151 (4), 264-269.

Moraes, E. B., Sanchez, M. C. O., Valente, G. S. C., Souza, D. F. \& Nassar, P. R. B. (2020). A segurança dos profissionais de saúde em tempos de COVID-19: uma reflexão. Research, Society and Development, 9 (7): 1-15, e134973832.

Norma Regulamentadora 32 (2005). Segurança e saúde no trabalho em serviços de saúde. Portaria $\mathrm{n}^{\circ} 485$, de 11 de novembro de 2005. http://www.fiocruz.br/biosseguranca/Bis/manuais/legislacao/NR-32.pdf.

Oliveira, W. S., Araujo, R. A. F. \& Migueis, G. S. (2020). Estresse em uma equipe de centro cirúrgico perante pandemia por Covid-19: um relato de experiência. Anais do I Congresso Norte Nordeste Saúde Pública (online): Editora Omnis Scientia. https://editoraomnisscientia.com.br/catalogos/ciencias-dasaude/anais-do-i-congresso-norte-nordeste-de-saude-publica-online-resumos-simples/. 
Research, Society and Development, v. 10, n. 6, e49310615867, 2021

(CC BY 4.0) | ISSN 2525-3409 | DOI: http://dx.doi.org/10.33448/rsd-v10i6.15867

Organização das Nações Unidas (ONU) (2020). Casos de Covid-19 estão se estabilizando no Brasil e nos EUA, segundo OPAS. https://news.un.org/pt/story/2020/09/1725132.

Ribeiro, A. P., Oliveira, G. L., Silva, L. S. \& Souza, E. R. (2020). Saúde e segurança de profissionais de saúde no atendimento a pacientes no contexto da pandemia de Covid-19: revisão de literatura. Rev. bras. saúde ocup., 45.

Santana, L. de L. (2018). Riscos psicossociais e saúde mental em ambiente hospitalar: com a voz o trabalhador. Universidade Federal do Paraná.

Silva, L. S., Machado, E. L., Oliveira, H. N. \& Ribeiro, A. P. (2020). Condições de trabalho e falta de informações sobre o impacto da COVID-19 entre trabalhadores da saúde. Rev. bras. saúde ocup., 45 (e24).

Silva, M. S. \& Migueis, G. S. (2020). Riscos enfrentados pela equipe de Enfermagem frente ao Coronavirus: uma revisão integrativa. Anais do I Congresso Norte Nordeste Saúde Pública (online): Editora Omnis Scientia. https://editoraomnisscientia.com.br/catalogos/ciencias-da-saude/anais-do-i-congresso-nortenordeste-de-saude-publica-online-resumos-simples/.

Soares, C. B., Hoga, L. A. K., Peduzzi, M., Sangaleti, C., Yonekurra, T. \& Silva, D. R. A. D. (2014). Revisão integrativa: conceitos e métodos utilizados na enfermagem. Rev. Esc. Enferm. USP, 48 (2), 335-345.

Soares, S. S. S., Souza, N. V. D. O., Carvalho, E. C., Varella, T. C. M. e M. L., Andrade, K. B. S., Pereira, S. E. M. et al (2020). De cuidador a paciente: na pandemia da Covid-19, quem defende e cuida da enfermagem brasileira? Esc. Anna Nery. 24.

Stillwell, S. B., Fineout-Overholt, E., Melnyk, B. M. \& Williamson, K. M. (2010). Asking the Clinical Question: A Key Step in Evidence-Based Practice. American Journal of Nursing, 110, 58-61.

Teixeira, C. F. S., Soares, C. M., Souza, E. A., Lisboa, E. S., Pinto, I. C. M., Andrade, L. R. et al (2020). A saúde dos profissionais de saúde no enfrentamento da pandemia de Covid-19. Ciênc. saúde coletiva, 25 (9), 3465-3474.

Wang, C., Pan, R., Wan, X., Tan, Y., Xu, L., Ho, C. S. et al (2020). Immediate psychological responses and associated factors during the initial stage of the 2019 Coronavirus Disease (COVID-19) epidemic among the general population in China. International Journal of Environmental Research and Public Health, 17 (5).

Whittemore, R. \& Knafl, K. (2005). The integrative review: updated methodology. J Adv Nurs., 52 (5), 546-53.

World Health Organization (WHO) (2020). Coronavírus disease (COVID-19). https://www.who.int/emergencies/diseases/novel-coronavirus-2019/questionand-answers-hub/q-a-detail/q-a-coronaviruses\#: : text=symptoms.

Zhan, Y., Wei, L., Li, H., Pan, Y., Wang, J., Li, Q. et al (2020). The process of psychological change of frontline nurses who care for patientes with COVID19 during their outbreak. 